\title{
Continuous EGFR tyrosine kinase inhibitor treatment with or without chemotherapy beyond gradual progression in non-small cell lung cancer patients
}

\author{
This article was published in the following Dove Press journal: \\ OncoTargets and Therapy \\ 28 August 2017 \\ Number of times this article has been viewed
}

\author{
Ling Peng \\ Yina Wang' \\ Yemin Tang' \\ Lei Zeng' \\ Junfang Liu' \\ Zhu Zeng' \\ Jian Liu' \\ Peng Shi ${ }^{2,3}$ \\ Xianghua $\mathrm{Ye}^{4}$ \\ Qiong Zhao' \\ 'Department of Thoracic Oncology, \\ The First Affiliated Hospital, School \\ of Medicine, Zhejiang University, \\ Hangzhou, ${ }^{2}$ Department of Medical \\ Statistics, Children's Hospital of \\ Fudan University, ${ }^{3}$ Center for \\ Evidence Based Medicine, Fudan \\ University, Shanghai, ${ }^{4}$ Department \\ of Radiotherapy, The First Affiliated \\ Hospital, School of Medicine, Zhejiang \\ University, Hangzhou, China
}

Correspondence: Qiong Zhao Department of Thoracic Oncology, The First Affiliated Hospital, School of Medicine, Zhejiang University, Hangzhou, Zhejiang Province, China

Tel/fax +86 57/ 87236800

Email doczq.2008@hotmail.com
Background: Several clinical studies have demonstrated that continuous administration of epidermal growth factor receptor (EGFR) tyrosine kinase inhibitor (TKI) could provide additional survival benefit for advanced non-small cell lung cancer (NSCLC) patients who had benefited from prior EGFR TKI therapy. However, whether EGFR TKI combined with chemotherapy could further prolong survival in patients with gradual progression is still unclear. The present study was conducted to evaluate the clinical outcome of continuous EGFR TKI treatment in combination with chemotherapy (combination group) versus continuous EGFR TKI treatment only (monotherapy group) in such a clinical setting.

Methods: We designed a cohort study to collect all chart data of NSCLC patients treated with EGFR TKI in our institution from February 2012 to December 2015 retrospectively and followed up the clinical outcome of EGFR TKI monotherapy or therapy in combination with chemotherapy until April 2017 prospectively. All eligible patients had to meet the criteria of gradual progression. The time interval of progression-free survival 1 (PFS1, gradual progression or death) to PFS2 (off-EGFR TKI progression), and overall survival (OS) between the above 2 groups were used in survival analysis.

Results: In all, 50 patients were included in our study. Patients' baseline characteristics were well balanced. Exon 19 deletion mutations and L858R point mutations were detected in 16 and 8 patients, respectively. Twenty, 22, and 8 patients were treated with EGFR TKI in the first, second, and third line setting, respectively. The time interval from PFS1 to PFS2 was 92 and 37 days (monotherapy vs combination), respectively (hazard ratio $[\mathrm{HR}]=1.16,95 \%$ confidence interval [CI]: $0.61-2.21, P=0.652$ ). The median OS in the monotherapy group and combination group was 696 and 799 days, respectively (HR $=0.74,95 \% \mathrm{CI}: 0.33-1.71, P=0.501$ ). There were no statistical differences between the 2 groups in terms of the time interval from PFS1 to PFS2 and OS.

Conclusion: Our results suggested that compared with EGFR TKI monotherapy, its combination with chemotherapy beyond gradual progression may not confer a significant survival benefit to NSCLC patients. Further prospective studies are warranted to reinforce the results of the study.

Keywords: epidermal growth factor receptor, tyrosine kinase inhibitor, non-small cell lung cancer

\section{Introduction}

Non-small cell lung cancer (NSCLC) is the leading cause of death from cancer for both men and women. NSCLC patients with epidermal growth factor receptor (EGFR) 
activating mutations significantly benefited from EGFR tyrosine kinase inhibitors (TKIs) as first line therapy, ${ }^{1,2}$ and second and third line therapy in terms of prolonged progression-free survival (PFS) ${ }^{3,4}$ However, all patients with EGFR-mutant lung cancer, who were initially responsive to EGFR TKI therapy, eventually become resistant to EGFR TKI treatment and experience disease progression. Acquired resistance to EGFR TKI has been defined by Jackman et al. ${ }^{5}$ Acquired resistance to TKIs usually develops within 1-2 years of therapy and represents a major challenge in the treatment for this subgroup of patients. There are different options for treatment beyond progression in EGFR mutation positive metastatic NSCLC, but the optimal strategy is still to be defined. The mechanism of acquired TKI resistance is related with a second mutation, for example, T790M, or c-MET amplification, whereas other manifestations of the tumor may still be sensitive to TKI therapy. ${ }^{6}$ Therefore, the paradigm to discontinue TKI upon progression and switch to chemotherapy may not be optimal for targeted therapies such as EGFR TKI. Instead, it may be reasonable to continue EGFR TKI beyond progression or add chemotherapy, radiotherapy, local surgery, or best supportive care, etc. It has been reported that continued treatment with EGFR TKI beyond progression benefits patients with activating EGFR mutations. ${ }^{7,8}$

Yang et al reported the complexity of causes of EGFR TKI failure and classified 3 clinical modes of EGFR TKI progression as dramatic progression, gradual progression, and local progression. ${ }^{9}$ Among them, the gradual progression group showed the longest disease control, progression-free survival, and most persistent symptom benefit. Gradual progression was defined as disease control $\geq 6$ months, compared with previous assessment, minor increment of tumor burden, and symptom score $\leq 1$. For that special group of patients, real-world data and clinical trials to demonstrate the optimal treatment choices are still lacking. We retrospectively collected clinical data regarding real-world studies on EGFR TKI with or without chemotherapy in NSCLC patients following EGFR TKI gradual progression.

\section{Patients and methods}

\section{Study population}

We analyzed all NSCLC patients treated with EGFR TKI (icotinib, erlotinib, and gefitinib) at our department from February 2012 to November 2015 who progressed after disease response (complete or partial remission or stable disease) on EGFR TKI for at least 6 months. The last follow-up was performed on April 30, 2017.

Patients met the following criteria to fall in the category of gradual progression, 1) disease control $\geq 6$ months, 2) compared with previous assessment, minor increment of tumor burden, and 3) symptom score $\leq 1 .{ }^{9}$ Tumors were subtyped histologically according to the World Health Organization classification. ${ }^{10}$ Computed tomography scans of thorax and upper abdomen were obtained within 14 days before start of TKI, and generally repeated every 6-12 weeks of treatment with TKI. Response was assessed using the Response Evaluation Criteria in Solid Tumors (RECIST) criteria ${ }^{11}$ in patients with at least one measurable lesion. The assessments were performed by each physician in charge. Toxicity was graded according to the Common Terminology Criteria for Adverse Events of the National Cancer Institute, version 4.0. The highest toxicity grade for each patient in all cycles of chemotherapy was used for the toxicity analysis. The Committee on Research Involving Human Subjects of Zhejiang University approved the study and waived the informed consent of patients due to the retrospective nature of the current study. This study is registered with ClinicalTrials.gov, number NCT01998061. Also, this study did not disclose the identity or private information of any of the study patients. The authors accept ethical obligation as members of medical profession to hold all information provided by patients and their families in strictest confidence.

\section{Study design}

PFS1 was defined as the time from commencement of EGFR TKI to the first documentation of progressive disease (PD) or death from any cause. PFS2 was defined as the time from commencement of EGFR TKI to off-TKI treatment. Overall survival (OS) was calculated from the commencement of TKI to the last visit or death from any cause. Treatment protocols fall into 1 of 2 categories: continuation of EGFR TKI or EGFR TKI with chemotherapy. The chemotherapy regimens were decided by the treating physicians.

Patients underwent a re-biopsy after the development of acquired resistance. Biopsies were carried out in the least invasive manner possible and typically consisted of lung or lymph node biopsy done with image guidance or rarely excisional biopsies. Histology was reviewed. Samples underwent genotyping for mutations in EGFR. Malignant effusions were collected to create cell blocks from which DNA was extracted. Plasma EGFR mutation was analyzed by digital droplet polymerase chain reaction (ddPCR).

\section{Statistical analysis}

Medical records were reviewed to obtain clinical information. The median time of PFS and OS was assessed with the Kaplan-Meier method. We estimated the hazard ratios (HRs) and corresponding 95\% confidence interval (CI) with the 
Cox proportional hazard regression model. We used SPSS (version 19.0; IBM Corporation, Armonk, NY, USA) for all statistical analyses and $P<0.05$ was considered statistically significant.

\section{Results}

\section{Patients' characteristics}

Fifty consecutive NSCLC patients treated with EGFR TKI who met the criteria of gradual progression were included in our retrospective study. Their clinicopathologic features are summarized in Table 1. Characteristics of patients among the 2 treatment groups were balanced regarding gender, age, smoking history, Eastern Cooperative Oncology Group performance status, EGFR mutation status, and line of TKI treatment. There were 22 men (44\%) and 28 women (56\%). All patients were diagnosed with stage IV disease

Table I Characteristics of patients

\begin{tabular}{|c|c|c|c|}
\hline Characteristics & $\begin{array}{l}\text { TKI } \\
n=36\end{array}$ & $\begin{array}{l}\text { TKI + chemo } \\
n=14\end{array}$ & $P$-value \\
\hline Sex & & & 0.919 \\
\hline Male & $16(44.4)$ & $6(42.9)$ & \\
\hline Female & $20(55.6)$ & $8(57.1)$ & \\
\hline Age, years & & & 0.295 \\
\hline Mean \pm SD & $57.39 \pm 12.34$ & $54.7 I \pm 5.50$ & \\
\hline Min, $\max$ & 36,83 & 47,66 & \\
\hline Histology & & & 0.186 \\
\hline Adenocarcinoma & $35(97.2)$ & $12(85.7)$ & \\
\hline Squamous & I (2.8) & $2(14.3)$ & \\
\hline Mutation status & & & 0.915 \\
\hline Exon 19 & $12(33.3)$ & $4(28.6)$ & \\
\hline L858R & $6(16.7)$ & $2(14.3)$ & \\
\hline Wide type & I (2.8) & I (7.I) & \\
\hline Unknown & $17(48.6)$ & $7(50.0)$ & \\
\hline Smoking & $14(38.9)$ & $4(28.6)$ & 0.495 \\
\hline Tumor burden & & & 0.540 \\
\hline I & I (2.8) & $0(0.0)$ & \\
\hline 2 & $18(50.0)$ & $5(35.7)$ & \\
\hline 3 & 17 (47.2) & $9(64.3)$ & \\
\hline TKI drug & & & 0.562 \\
\hline First line & 14 (38.9) & $6(42.9)$ & \\
\hline Second line & $15(4 \mid .7)$ & $7(50.0)$ & \\
\hline Third line & $7(19.4)$ & I (7.I) & \\
\hline Type of TKI & & & 0.542 \\
\hline Erlotinib & 4 (II.I) & $2(14.3)$ & \\
\hline Gefitinib & $2(5.6)$ & $2(14.3)$ & \\
\hline Icotinib & $30(83.3)$ & $10(71.4)$ & \\
\hline \multicolumn{3}{|c|}{ TKI treatment duration (days) } & 0.029 \\
\hline Median $\left(\mathrm{P}_{25}, \mathrm{P}_{75}\right)$ & $482(352,605)$ & $374(269,438)$ & \\
\hline Min, $\max$ & $212,1,427$ & 182,855 & \\
\hline
\end{tabular}

Notes: Data were expressed as mean \pm SD or median $\left(P_{25}, P_{75}\right)$ for continuous variables with normal or non-normal distribution and compared between groups using the independent Student's $t$-test or Mann-Whitney $U$ test accordingly. Categorical variables were expressed as $n(\%)$ and compared between groups using Pearson's chi-square test or Fisher's exact test when appropriate.

Abbreviations: $P_{25}$, the 25 th percentile; $P_{75}$, the 75th percentile; SD, standard deviation; TKI, tyrosine kinase inhibitor. upon commencement of EGFR TKI. The most common histologic subtype was adenocarcinoma (94\%), and adequate tumor samples for analysis of gene status were obtained in 26 patients. Exon 19 deletion mutations and L858R point mutations were detected in 16 and 8 patients, respectively. Forty percent of patients received first line EGFR TKI.

\section{Clinical outcome}

Survival data were analyzed and are summarized in Table 2. Median follow-up was 1,287 (range 873-1,915) days. The median PFS1 (RECIST progression) of patients receiving EGFR TKI alone was 360 days, while in the combination group, it was 262 days; there was a statistically significant difference between the arms ( $\mathrm{HR}=2.12,95 \% \mathrm{CI}: 1.10-4.07$, $P=0.024$ ) (Figure 1). The median PFS2 was 481 and 395 days (monotherapy vs combination), respectively ( $\mathrm{HR}=1.85,95 \%$ CI: $0.97-3.51, P=0.060$ ) (Figure 2). The time interval from PFS1 to PFS2 was 92 and 37 days (monotherapy vs combination), respectively ( $\mathrm{HR}=1.16,95 \% \mathrm{CI}: 0.61-2.21, P=0.652$ ) (Figure 3). The median OS in the monotherapy group and combination group was 696 and 799 days, respectively (HR $=0.74,95 \%$ CI: $0.33-1.71, P=0.501$ ) (Figure 4). There were no statistical differences between the 2 groups in terms of the time interval from PFS1 to PFS2 and OS.

We also performed a subgroup analysis according to EGFR mutation status. The results are shown in Table 3. Time interval from PFS1 to PFS2 was significantly higher in the monotherapy group than the combination group in EGFR status unknown patients $(P=0.009)$, but there was no significance in OS $(P=0.754)$. No significances were observed in other subgroups.

\section{Findings at the time of acquired resistance} Eleven patients had biopsy samples that were sufficient for molecular analysis, including fine needle aspirations, core biopsies, and cytology from malignant effusions, of which 7 patients were in the combination group, while 4 patients were in the monotherapy group. All specimens underwent pathologic review by a thoracic pathologist. Six patients were tested for their serum EGFR mutation status by ddPCR, of which 5 and 1 were in the combination and monotherapy groups, respectively. We identified a second-site EGFR T790M mutation in 11 of 17 samples. There were no serious adverse events related with the biopsies.

\section{Toxicity profiles of EGFR TKI with or without chemotherapy}

To determine whether EGFR TKI with chemotherapy caused more adverse events, we assessed the toxicity profiles of all 
Table 2 Prognosis of treatment

\begin{tabular}{|c|c|c|c|c|c|c|c|}
\hline \multirow{2}{*}{$\begin{array}{l}\text { Survival } \\
\text { characteristics }\end{array}$} & \multirow{2}{*}{$\begin{array}{l}\text { TKI } \\
n=36\end{array}$} & \multirow{2}{*}{$\begin{array}{l}\text { TKI + chemo } \\
n=14\end{array}$} & \multicolumn{3}{|c|}{ TKI + chemo vs TKI } & \multicolumn{2}{|c|}{ Log-rank test } \\
\hline & & & HR & $95 \% \mathrm{Cl}$ & $P$-value & $\overline{x^{2}}$ & $P$-value \\
\hline \multicolumn{8}{|l|}{ PFSI (days) } \\
\hline Median $\left(\mathrm{P}_{25}, \mathrm{P}_{75}\right)$ & $360(250,482)$ & $262(226,364)$ & 2.12 & $1.10-4.07$ & 0.024 & 5.318 & 0.021 \\
\hline PFS2 (days) & & & & & & & \\
\hline $\begin{array}{l}\text { Median }\left(\mathrm{P}_{25}, \mathrm{P}_{75}\right) \\
\text { PFSI to PFS2 (days) }\end{array}$ & $48 I(352,566)$ & $395(277,460)$ & 1.85 & $0.97-3.51$ & 0.060 & 3.655 & 0.056 \\
\hline $\begin{array}{l}\text { Median }\left(P_{25}, P_{75}\right) \\
\text { OS (days) }\end{array}$ & $92(54,196)$ & $37(27,156)$ & 1.16 & $0.6 \mathrm{I}-2.2 \mathrm{I}$ & 0.652 & 0.205 & 0.650 \\
\hline Median $\left(\mathrm{P}_{25}, \mathrm{P}_{75}\right)$ & $696(503,98 \mathrm{I})$ & $799(618,911)$ & 0.76 & $0.33-1.71$ & 0.501 & 0.457 & 0.499 \\
\hline
\end{tabular}

Notes: We assessed the median time of progression-free survival and overall survival with the Kaplan-Meier method. We estimated the $\mathrm{HRs}$ and corresponding $95 \% \mathrm{Cl}$ values with the Cox proportional hazard regression model. We used SPSS (version 19.0; IBM Corp., Armonk. NY, USA) for all statistical analyses and $P<0.05$ was considered statistically significant.

Abbreviations: $\mathrm{P}_{25}$, the 25 th percentile; $\mathrm{P}_{75}$, the 75 th percentile; $\mathrm{HR}$, hazard ratio, $\mathrm{Cl}$, confidence interval; SD, standard deviation; TKI, tyrosine kinase inhibitor; OS, overall survival; PFS, progression-free survival; chemo, chemotherapy.

patients. Table 4 lists the incidence of hematological and non-hematological toxicities of all grades. In the present study, severe hematologic toxicity was observed with a higher incidence in the combination group. Grade 3 or 4 hematologic toxicity of neutropenia was observed in 5 out of 14 patients (35.8\%) from the combination group, but was absent in the monotherapy group. Other grade $3 / 4$ hematological toxicities in the combination group were anemia ( $7.1 \%, 1$ of 14 patients). Grade 3 non-hematological toxicities were anorexia (14.2\%, 2 of 14), fatigue ( $28.6 \%, 4$ of 14$)$, nausea (14.2\%, 2 of 14$)$, and vomiting (7.1\%, 1 of 14). No grade 4 non-hematological toxicities were observed. No treatment-related deaths occurred. None of the patients manifested interstitial lung disease in response to EGFR TKI. The tolerability profile reported in this study was generally consistent with the known safety profile of TKI and chemotherapy and showed no new adverse events.

\section{Discussion}

EGFR-mutant NSCLC is sensitive to EGFR TKIs. The administration of TKIs beyond progression is becoming

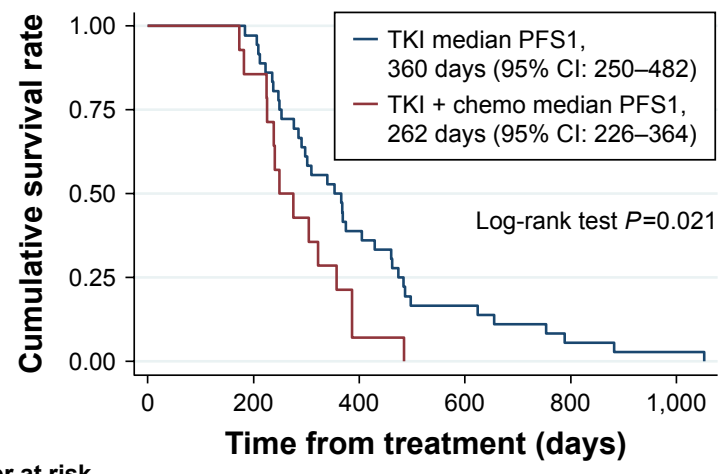

Number at risk

$\begin{array}{lllllll}\text { TKI group } & 36 & 35 & 14 & 6 & 2 & 1 \\ \text { TKI + chemo group } & 14 & 12 & 1 & 0 & 0 & 0\end{array}$

Figure I Survival curve of PFSI between TKI and TKI + chemo.

Abbreviations: $\mathrm{Cl}$, confidence interval; TKI, tyrosine kinase inhibitor; PFS, progression-free survival; chemo, chemotherapy. increasingly common clinical practice in patients with indolent and asymptomatic tumor growth, who may potentially continue to benefit from continuous EGFR TKI treatment. EGFR TKI continuation is consistent with preclinical data that continued EGFR inhibition is indicated due to the presence of a heterogeneous population of tumor cells, with varied sensitivity to EGFR inhibition, ${ }^{12}$ as well as clinical data showing disease flare after discontinuation of EGFR TKI. ${ }^{13,14}$ Because of the slow growth of resistant clones, discontinuation of TKI may paradoxically worsen the disease. ${ }^{14}$ A previous study suggested EGFR addiction persists after development of TKI resistance, prompting many clinicians to continue TKI along with chemotherapy. ${ }^{15}$ However, this strategy has not been formally evaluated.

Some clinicians have expressed concern that TKI plus chemotherapy could be antagonistic when administered without pharmacodynamic separation. Indeed, four large trials investigating the use of standard chemotherapy in combination with EGFR inhibitor treatment in the first line setting found that, although tolerable, that does not lead to

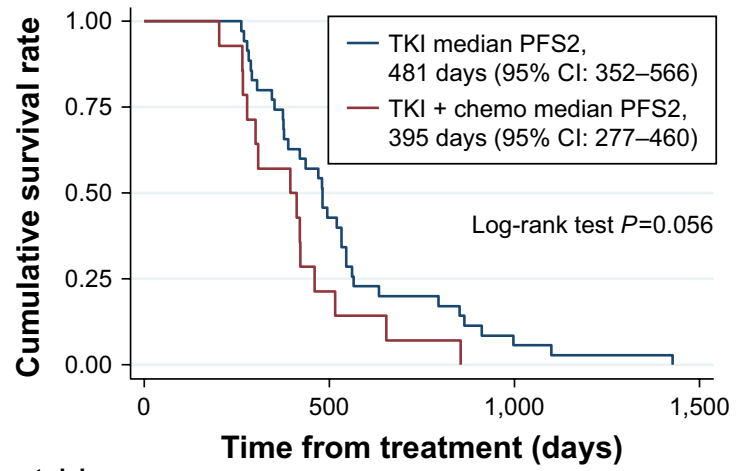

Number at risk

$\begin{array}{lllll}\text { TKI group } & 36 & 15 & 2 & 0 \\ \text { TKI + chemo group } 14 & 3 & 0 & 0\end{array}$

Figure 2 Survival curve of PFS2 between TKI and TKI + chemo.

Abbreviations: $\mathrm{Cl}$, confidence interval; TKI, tyrosine kinase inhibitor; PFS, progression-free survival; chemo, chemotherapy. 


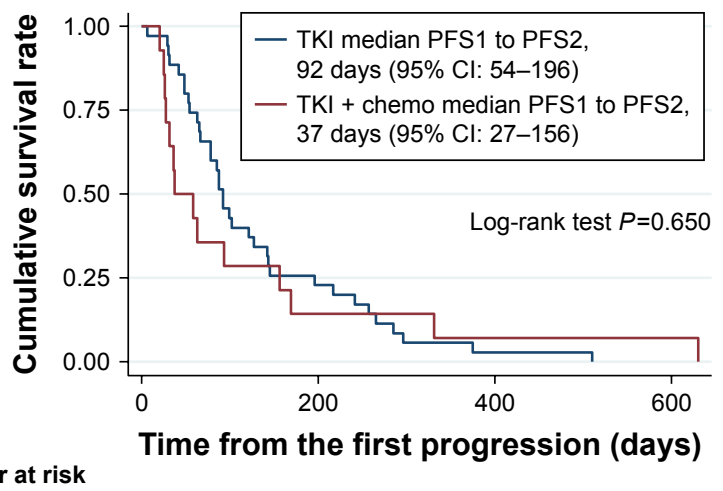

Number at risk

$\begin{array}{lllll}\text { TKI group } & 36 & 8 & 1 & 0 \\ \text { TKI + chemo group } & 14 & 2 & 1 & 1\end{array}$

Figure 3 Survival curve of PFSI to PFS2 between TKI and TKI + chemo. Abbreviations: $\mathrm{Cl}$, confidence interval; TKI, tyrosine kinase inhibitor; PFS, progression-free survival; chemo, chemotherapy.

any survival advantage. ${ }^{16-19}$ However, such combination treatment is not necessarily ineffective at progression since the underlying biology of the disease may have changed. Results of the IMPRESS trial found no statistically significant differences in median PFS and objective response between patients with acquired resistance to first line gefitinib who continued the same TKI after disease progression together with platinum doublet chemotherapy and those who received only chemotherapy. ${ }^{20}$ However, it does not necessarily follow that combination treatment will be ineffective at PD since the included patient cohort has been altered as gradual progression.

In clinical trials, treatment often stops at the time of RECIST progression. However, RECIST criteria could not reflect the diversity of failure in patients with EGFR TKI treatment. These criteria have been challenged in the EGFR TKI setting, since progression after EGFR TKI treatment seems to be different from what we observe in relapse after

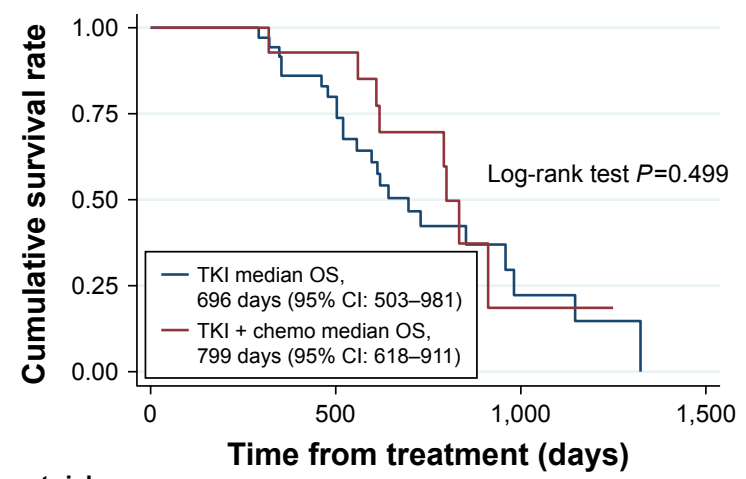

\section{Number at risk}

$\begin{array}{lllll}\text { TKI group } & 36 & 26 & 3 & 0\end{array}$

TKI + chemo group 14

12

0

Figure 4 Survival curve of OS between TKI and TKI + chemo.

Abbreviations: $\mathrm{Cl}$, confidence interval; $\mathrm{TKI}$, tyrosine kinase inhibitor; chemo, chemotherapy; OS, overall survival.
Table 3 Subgroup analysis according to EGFR mutation status

\begin{tabular}{llll}
\hline $\begin{array}{l}\text { Survival } \\
\text { characteristics }\end{array}$ & $\begin{array}{l}\text { EGFR mutation } \\
\mathbf{n}=\mathbf{2 6}\end{array}$ & $\begin{array}{l}\text { No EGFR mutation } \\
\mathbf{n = 2 4}\end{array}$ & P-value \\
\hline PFSI (days) & $276(223,369)$ & $368(275,484)$ & 0.032 \\
PFS2 (days) & $389(288,545)$ & $470(378,545)$ & 0.075 \\
PFSI to PFS2 (days) & $87(53,143)$ & $85(31,156)$ & 0.955 \\
OS (days) & $729(520,911)$ & $792(609,1,323)$ & 0.170 \\
\hline
\end{tabular}

Notes: The median time of progression-free survival and overall survival with the Kaplan-Meier method was assessed. Data were expressed as median (P25, P75). We used SPSS (version 19.0; IBM Corp., Armonk, NY, USA) for all statistical analyses and $P<0.05$ was considered statistically significant.

Abbreviations: EGFR, epidermal growth factor receptor; OS, overall survival; PFS, progression-free survival.

chemotherapy. Therefore, we adopted the concept of PFS1 and PFS2 as previously stated in the ASPIRATION trial, which demonstrated that first line erlotinib beyond progression is feasible and may delay salvage therapy in selected patients with EGFR mutation positive NSCLC. ${ }^{21}$ In our study, the monotherapy group delayed clinical progression (time interval from PFS1 to PFS2) for about 3 months, which is consistent with previously published data on PFS of EGFR TKI therapy. Interestingly, although not statistically significant, PFS1 was longer in patients who received EGFR TKI monotherapy than patients who received EGFR TKI plus chemotherapy. It suggests that patients with more indolent tumor received EGFR TKI monotherapy, which might be a potential bias influencing findings in the present study. Due to the small sample size of our study, selection bias or sampling error may exist.

In our study, reflecting real-world clinical practice, primary EGFR mutation status was not determined before the

Table 4 Toxicity profile in all treatment courses

\begin{tabular}{|c|c|c|c|c|c|c|}
\hline \multirow[t]{3}{*}{ Type of toxicity } & \multirow{2}{*}{\multicolumn{3}{|c|}{$\begin{array}{l}\text { TKI } \\
\text { Toxicity grade } \\
(\%, n=36)\end{array}$}} & \multirow{2}{*}{\multicolumn{3}{|c|}{$\begin{array}{l}\text { TKI + chemo } \\
\text { Toxicity grade } \\
(\%, n=I 4)\end{array}$}} \\
\hline & & & & & & \\
\hline & $\mathrm{I}-2$ & 3 & 4 & I-2 & 3 & 4 \\
\hline \multicolumn{7}{|l|}{ Hematological } \\
\hline Neutropenia & II.I & 0 & 0 & 71.4 & 35.8 & 0 \\
\hline Anemia & 16.7 & 0 & 0 & 42.8 & 7.1 & 0 \\
\hline Thrombocytopenia & 5.5 & 0 & 0 & 14.3 & 0 & 0 \\
\hline \multicolumn{7}{|l|}{ Non-hematological } \\
\hline Weight loss & 25 & 0 & 0 & 57.1 & 7.1 & 0 \\
\hline Anorexia & 13.9 & 0 & 0 & 42.9 & 14.3 & 0 \\
\hline Fatigue & 25 & 0 & 0 & 42.9 & 28.6 & 0 \\
\hline ALT/AST & 27.8 & 11.1 & 0 & 21.4 & 0 & 0 \\
\hline Nausea & 13.9 & 0 & 0 & 50 & 14.2 & 0 \\
\hline Vomiting & 5.6 & 0 & 0 & 35.8 & 7.1 & 0 \\
\hline Diarrhea & 33.3 & 0 & 0 & 28.6 & 0 & 0 \\
\hline Mucositis & 38.9 & 0 & 0 & 35.8 & 0 & 0 \\
\hline Skin rash & 30.6 & 8.3 & 0 & 42.9 & 28.6 & 0 \\
\hline
\end{tabular}

Note: Numbers denote the highest grade in each patient.

Abbreviations: ALT, alanine aminotransferase; AST, aspartate aminotransferase; TKI, tyrosine kinase inhibitor; chemo, chemotherapy. 
commencement of EGFR TKI treatment for all patients, since routine testing is currently not covered by medical insurance in China. Some of the patients, especially those on second or third line treatment, received EGFR TKI according to their clinical characteristics. Although not all patients in this study had EGFR testing, strict study eligibility and medical records review led to a highly enriched patient population. The subgroup analysis according to EGFR status revealed a significant difference in time interval from PFS1 to PFS2 in patients with EGFR status unknown. Considering the small number in each group, the result should be explained with caution.

The mechanisms of acquired resistance can be divided into 3 categories: genetic alterations in EGFR, secondary mutations, and target gene amplification. The most commonly observed mechanism of resistance is EGFR T790M, while acquired mutations in EGFR other than T790M, including T854A, D761Y, and L747S, are infrequent. ${ }^{22-24}$ Sufficient tissue from re-biopsies was not obtained for all the molecular tests currently conducted. Although resistance acquired through the T790M mutation may follow a more indolent course than clinical resistance without the mutation, we did not have adequate information regarding the progression mechanism or the resistance to EGFR TKI due to the limited number of patients undergoing re-biopsies.

Eleven patients harbored T790M mutation as the acquired resistance mechanism; however, third generation EGFR TKI drugs were not available at the time of the study. Consequently, the therapeutic choice was limited to TKI continuation, switch to chemotherapy, or TKI continuation in combination with chemotherapy. According to the subgroup analysis of the IMPRESS study, for plasma T790M-negative patients, the continuation of TKI in combination with platinum-based doublet chemotherapy may potentially offer some clinical benefit, but this requires further confirmation. ${ }^{20}$

The present study possesses several intrinsic limitations. Firstly, our study was an observational one. Although the characteristics of the patients at baseline were well balanced, the potential confounding factors cannot be all adjusted in a non-randomized study. Secondly, the sample size in our study was small and the number of patients in each group was unbalanced (36 patients in TKI group vs 14 patients in the TKI + chemo group), which may lead to insufficient statistical test power. Thirdly, we could not collect sufficient information of gene mutation status for this observational study. EGFR mutation status was unknown in $48 \%$ of patients, and only $22 \%$ of patients underwent re-biopsy or detection of plasma EGFR mutation by ddPCR. Finally, the physicians' choice of monotherapy versus combination was not randomized. Furthermore, the chemotherapy regimens used in the combination group were not predetermined. Given the above limitations, randomized trials evaluating the role of continuing EGFR TKI with or without chemotherapy beyond gradual progression are still needed.

In conclusion, our study showed no benefit of adding chemotherapy to continuing EGFR TKI monotherapy beyond gradual progression. Consistent with findings from other studies, our results did not support the practice of routinely continuing EGFR TKI in combination with chemotherapy in this setting.

\section{Acknowledgments}

This study was partially supported by a grant from the National Natural Science Foundation of China (no 81402179). A synopsis of the current study was presented as an abstract at the 2016 ASCO annual meeting (abstract no e20531).

\section{Author contributions}

All authors participated in the design of the study and contributed to manuscript development. Data were collected and interpreted in collaboration with all authors. All the authors vouch for the accuracy and completeness of the data reported and the adherence of the study to the protocol.

\section{Disclosure}

The authors report no conflicts of interest in this work.

\section{References}

1. Mok TS, Wu YL, Thongprasert S, et al. Gefitinib or carboplatinpaclitaxel in pulmonary adenocarcinoma. N Engl J Med. 2009;361(10): 947-957.

2. Zhou $\mathrm{C}, \mathrm{Wu}$ YL, Chen G, et al. Erlotinib versus chemotherapy as first-line treatment for patients with advanced EGFR mutation-positive non-small-cell lung cancer (OPTIMAL, CTONG-0802): a multicentre, open-label, randomised, phase 3 study. Lancet Oncol. 2011;12(8): 735-742.

3. Shepherd FA, Rodrigues Pereira J, Ciuleanu T, et al. Erlotinib in previously treated non-small-cell lung cancer. $N$ Engl J Med. 2005;353(2): $123-132$.

4. Kim ES, Hirsh V, Mok T, et al. Gefitinib versus docetaxel in previously treated non-small-cell lung cancer (INTEREST): a randomised phase III trial. Lancet. 2008;372(9652):1809-1818.

5. Jackman D, Pao W, Riely GJ, et al. Clinical definition of acquired resistance to epidermal growth factor receptor tyrosine kinase inhibitors in non-small-cell lung cancer. J Clin Oncol. 2010;28(2):357-360.

6. Sequist LV, Waltman BA, Dias-Santagata D, et al. Genotypic and histological evolution of lung cancers acquiring resistance to EGFR inhibitors. Sci Transl Med. 2011;3(75):75ra26.

7. Asami K, Okuma T, Hirashima T, et al. Continued treatment with gefitinib beyond progressive disease benefits patients with activating EGFR mutations. Lung Cancer. 2013;79(3):276-282. 
8. Faehling M, Eckert R, Kamp T, et al. EGFR-tyrosine kinase inhibitor treatment beyond progression in long-term Caucasian responders to erlotinib in advanced non-small cell lung cancer: a case-control study of overall survival. Lung Cancer. 2013;80(3):306-312.

9. Yang JJ, Chen HJ, Yan HH, et al. Clinical modes of EGFR tyrosine kinase inhibitor failure and subsequent management in advanced nonsmall cell lung cancer. Lung Cancer. 2013;79(1):33-39.

10. Beasley MB, Brambilla E, Travis WD. The 2004 World Health Organization classification of lung tumors. Semin Roentgenol. 2005; 40(2):90-97.

11. Therasse P, Arbuck SG, Eisenhauer EA, et al. New guidelines to evaluate the response to treatment in solid tumors. European Organization for Research and Treatment of Cancer, National Cancer Institute of the United States, National Cancer Institute of Canada. J Natl Cancer Inst 2000;92(3):205-216.

12. Chmielecki J, Foo J, Oxnard GR, et al. Optimization of dosing for EGFR-mutant non-small cell lung cancer with evolutionary cancer modeling. Sci Transl Med. 2011;3(90):90ra59.

13. Riely GJ, Kris MG, Zhao B, et al. Prospective assessment of discontinuation and reinitiation of erlotinib or gefitinib in patients with acquired resistance to erlotinib or gefitinib followed by the addition of everolimus. Clin Cancer Res. 2007;13(17):5150-5155.

14. Chaft JE, Oxnard GR, Sima CS, Kris MG, Miller VA, Riely GJ. Disease flare after tyrosine kinase inhibitor discontinuation in patients with EGFR-mutant lung cancer and acquired resistance to erlotinib or gefitinib: implications for clinical trial design. Clin Cancer Res. 2011; 17(19):6298-6303.

15. Goldberg SB, Oxnard GR, Digumarthy S, et al. Chemotherapy with erlotinib or chemotherapy alone in advanced non-small cell lung cancer with acquired resistance to EGFR tyrosine kinase inhibitors. Oncologist. 2013;18(11):1214-1220.

16. Giaccone G, Herbst RS, Manegold C, et al. Gefitinib in combination with gemcitabine and cisplatin in advanced non-small-cell lung cancer: a phase III trial - INTACT 1. J Clin Oncol. 2004;22(5):777-784.
17. Herbst RS, Giaccone G, Schiller JH, et al. Gefitinib in combination with paclitaxel and carboplatin in advanced non-small-cell lung cancer: a phase III trial - INTACT 2. J Clin Oncol. 2004;22(5):785-794.

18. Herbst RS, Prager D, Hermann R, et al. TRIBUTE: a phase III trial of erlotinib hydrochloride (OSI-774) combined with carboplatin and paclitaxel chemotherapy in advanced non-small-cell lung cancer. J Clin Oncol. 2005;23(25):5892-5899.

19. Gatzemeier U, Pluzanska A, Szczesna A, et al. Phase III study of erlotinib in combination with cisplatin and gemcitabine in advanced non-small-cell lung cancer: the Tarceva Lung Cancer Investigation Trial. J Clin Oncol. 2007;25(12):1545-1552.

20. Soria JC, Wu YL, Nakagawa K, et al. Gefitinib plus chemotherapy versus placebo plus chemotherapy in EGFR-mutation-positive non-small-cell lung cancer after progression on first-line gefitinib (IMPRESS): a phase 3 randomised trial. Lancet Oncol. 2015;16(8):990-998.

21. Park K, Yu CJ, Kim SW, et al. First-line erlotinib therapy until and beyond response evaluation criteria in solid tumors progression in Asian patients with epidermal growth factor receptor mutation-positive non-small-cell lung cancer: the ASPIRATION study. JAMA Oncol. 2016;2(3):305-312.

22. Balak MN, Gong Y, Riely GJ, et al. Novel D761Y and common secondary T790M mutations in epidermal growth factor receptor-mutant lung adenocarcinomas with acquired resistance to kinase inhibitors. Clin Cancer Res. 2006;12(21):6494-6501.

23. Costa DB, Halmos B, Kumar A, et al. BIM mediates EGFR tyrosine kinase inhibitor-induced apoptosis in lung cancers with oncogenic EGFR mutations. PLoS Med. 2007;4(10):1669-1679.

24. Bean J, Riely GJ, Balak M, et al. Acquired resistance to epidermal growth factor receptor kinase inhibitors associated with a novel T854A mutation in a patient with EGFR-mutant lung adenocarcinoma. Clin Cancer Res. 2008;14(22):7519-7525.
OncoTargets and Therapy

\section{Publish your work in this journal}

OncoTargets and Therapy is an international, peer-reviewed, open access journal focusing on the pathological basis of all cancers, potential targets for therapy and treatment protocols employed to improve the management of cancer patients. The journal also focuses on the impact of management programs and new therapeutic agents and protocols on

\section{Dovepress}

patient perspectives such as quality of life, adherence and satisfaction. The manuscript management system is completely online and includes a very quick and fair peer-review system, which is all easy to use. Visit http://www.dovepress.com/testimonials.php to read real quotes from published authors. 